\title{
Analysis and realization of synchronized swimming in URWPGSim2D
}

\author{
Han Lu ${ }^{1, a^{*}}$, Li Shu-qin ${ }^{2, b^{*}}$ \\ ${ }^{1}$ School of computer, Beijing Information Science\& Technology University, Beijing, P.R. China, \\ ${ }^{2}$ School of computer, Beijing Information Science\& Technology University, Beijing, P.R. China, \\ a ${ }^{*}$ hanlu1159245@163.com, ${ }^{\text {* }}$ lishuqin_de@126.com
}

Keywords: underwater robot; robot fish; synchronized swimming.

Abstract. Hosted by the International Robot Alliance, the international water robot contest has held the 8th so far. There are big changes in rules in the synchronized swimming events of the URWPGSim2D simulation platform, the original rule changes from no restrictions by the performance content to rule with standard combination of movement and freedom of action. This paper mainly focuses on the latest competition rules, and gives the implementation strategy of the corresponding action. The strategy implementation was awarded first prize in the international water robot contest in 2015, which shows the effectiveness of the strategy.

\section{Introduction}

The main content of international water robot competition is to make intelligent bionic robot fish racing in water, synchronized swimming, chasing heated confrontation, etc. The tournament has a strong technical challenges and high artistic ornamental, it is the perfect combination of research and science. This competition is mainly divided into two types of projects, including physical machine fish and 2D simulation robotic fish. The content of the 2D simulation project change from the original water polo 5V5, ball big battle, snooker water polo, synchronized swimming, collaboration vias, to the ball game, survival challenge, synchronized swimming and water transport now.

$2 \mathrm{D}$ simulation synchronized swimming robot uses water polo game $2 \mathrm{D}$ version of the software as the game platform. Events adopt standard simulation site $(3000 \mathrm{~mm} * 2000 \mathrm{~mm}), 1.5$ times the size of the venue, specifications $4500 \mathrm{~mm} * 3000 \mathrm{~mm}$. Emulator server side is used to simulate the water environment, showing the game process, and the client is used to load simulation game strategy.

According to the rules of 2015 2D simulation synchronized swimming, each school have one team, each team has 10 simulation robotic fish, including 1 yellow fish and 9 red fish. The initial state is a team of 10 simulation robotic fish, which location and direction are randomly distributed in the simulation field. After the start of the game, the 1 simulation machine fish swimming in the playing area randomly. The other 9 simulation machines are controlled by the participants, cooperate with the 1st one to perform. The game time is 5 minutes, in which the standard action is 3 and free action is 2.The game only once, during the race there shall not be suspended. According to the team's performance of smooth, creative, beautiful shape, whether fit the subject and so on, the referee while give a score. The biggest change in rules is the addition of standard action, include an Arabic numerals shape, a closed geometry, 5 seconds between two styling, and keeping all the same shape and movement more than five seconds.

The change of the rules is a rebirth of the project, so this article mainly analyzes and studies the four standard action of the new competition rules, and gives the corresponding implementation strategy.

\section{Implementation of Arabia digital modeling}

\section{Introduction of Arabia digital modeling rules}

Competition rules: The Arabic numeral modeling can be a single digits or more numerals. Due to there are nine fish can be controlled, if the number of fish you put into Arabic Numbers is less than 9, 
then other fish modelling will be a problem at this time. Thus, team members typically utilize nine fish to put into the desired shape. Fig. 1 lists several digits modeling.

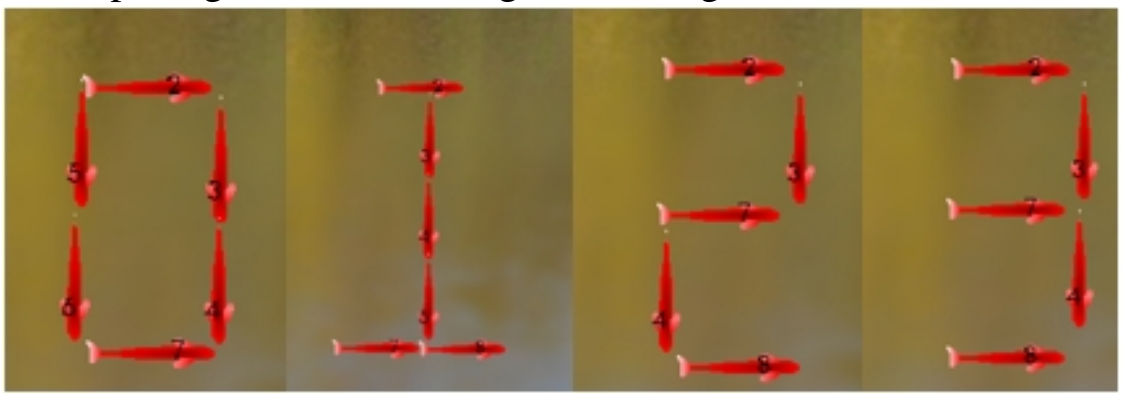

Fig. 1 several digits modeling

\section{Implementation of Arabic numerals}

The main idea of Arabic numerals policy implementation are: (1) Determine whether the fish to reach the target point. (2)Determine whether the fish reaches the target angle.

At present, the control parameters of the robot fish include: the position of the fish, the direction of the fish, the angular velocity of the fish and the line speed. Simulation platform control robotic fish linear and angular velocities by different gear. Angular gear is from 0 to 15, a total of 16 gear, 0 is the biggest left angular velocity, 7 is straight, 15 is the biggest turn right angular velocity. The fish Angle range is $(-\mathrm{PI}, \mathrm{PI}$.

If the fish is in the position of Point $1=(\mathrm{X} 1, \mathrm{Z} 1)$, the current moment of arc is $\mathrm{V} 1$, and the target point is Point $=(\mathrm{X}, \mathrm{Z})$, the target arc is V. The algorithm to achieve Arabia is as follows:

Step 1: Determine whether the fish reached the target point.

Step2: If the fish do not point to the target, calling Dribbleexptop () function, then the fish will swim to the target point. Dribbleexptop () function parameter list is as follows: Dribbleexptop (ref Decision decision, RoboFish fish, float angleTheta1, float angleTheta2, float disThreshold, int VCode1, int VCode2, int cycles, int msPerCycle, bool flag, xna.Vector3 GoalPoint), the function is to control certain fish to swim to a certain point.

Step3: If the target point is reached, determine whether reached the target Angle.

Step4: If the fish do not reach the target angle, then angle adjustment. If the fish is in radians (V, V $+\pi$ ) range, then the angular velocity is set to the maximum left turn TCode $=0$,else if the current in radians $(\mathrm{V}-\pi, \mathrm{V})$, then the angular velocity is set to the maximum right turn TCode $=15$.

Step5: Determine whether the fish rad is in $(\mathrm{V}-0.1, \mathrm{~V}+0.1)$, if not, then return to Step4, else set the fish completed the target action.

Fig.2-1 to Fig.2-4 shows the process and results of the program to achieve the Arabia digital 2.

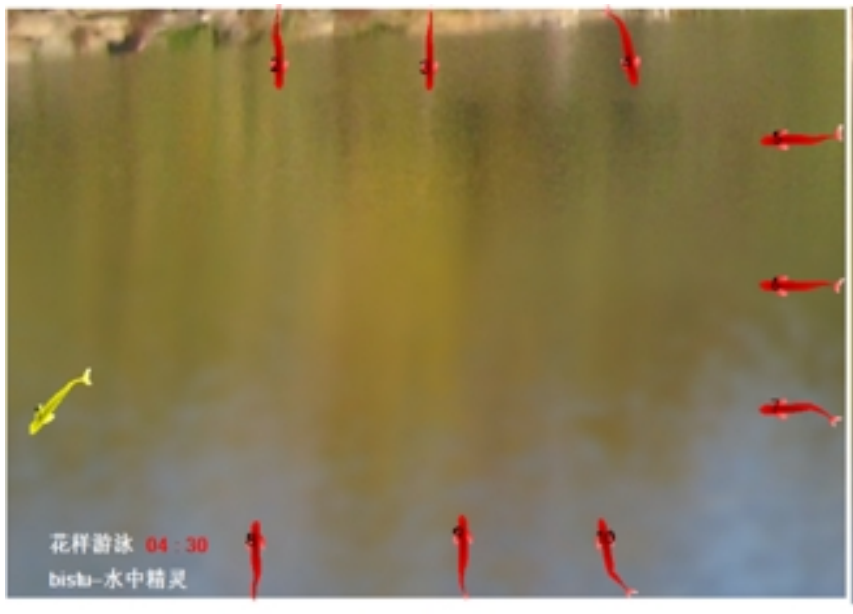

Fig.2-1 04:30 moment diagram

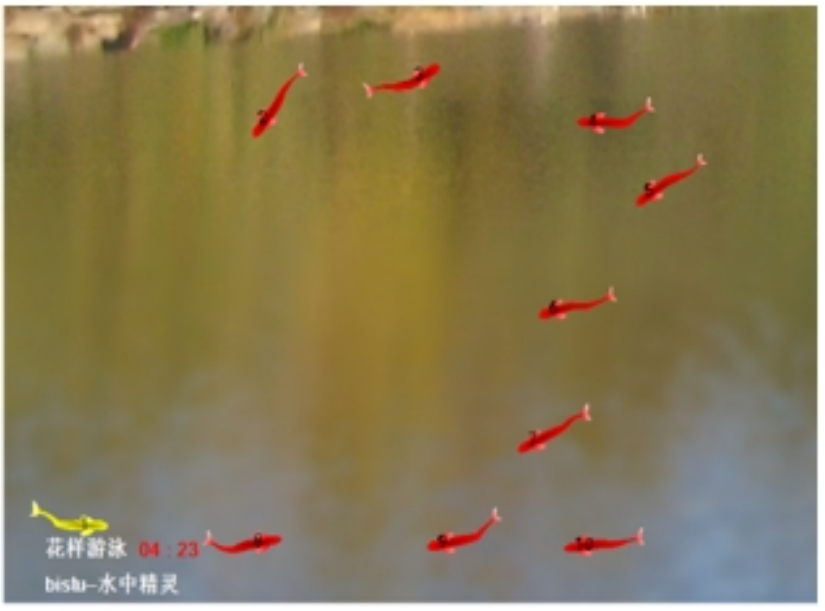

Fig.2-2 04:23 moment diagram 


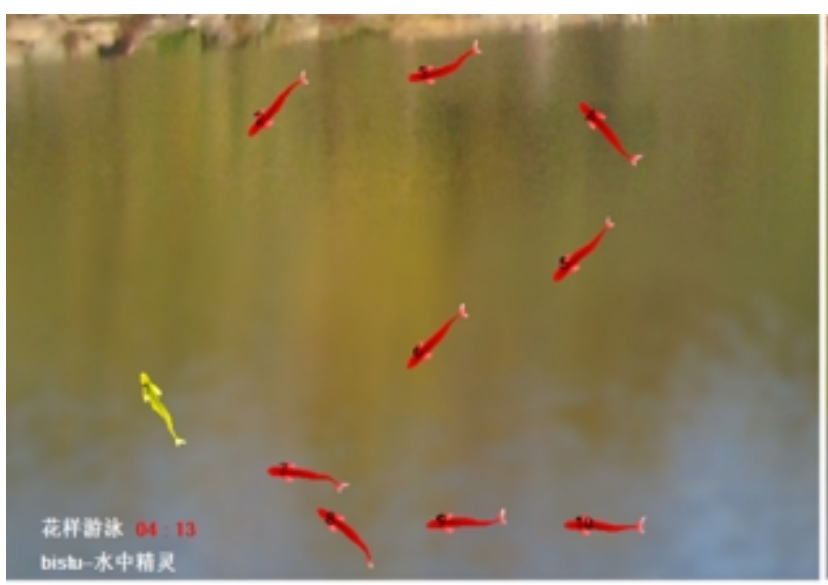

Fig.2-3 04:13 moment diagram

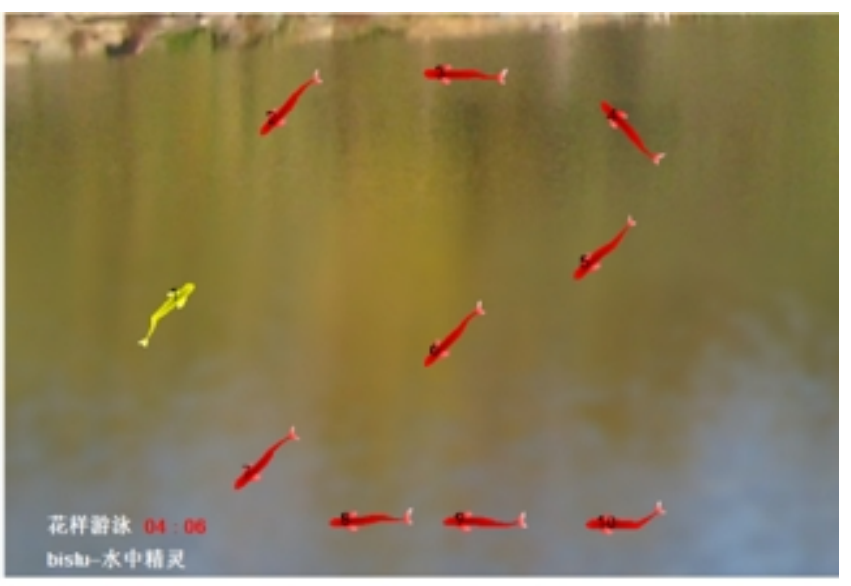

Fig.2-4 04:00 moment diagram

\section{Implementation of enclosed geometry}

\section{Introduction of enclosed geometry rules}

The rules of the game require the completion of a closed geometry pattern. The author thinks that the closed geometry of the rule can be set to a circle, triangle, square, diamond, etc. as shown in Fig.3. Of course, the more complex geometry of the more difficult, the higher the score.

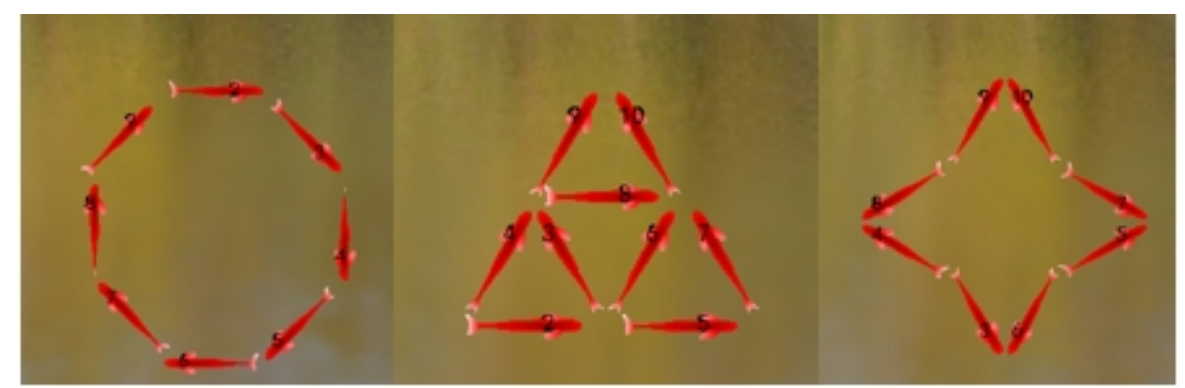

Fig.3 several geometric modeling

\section{Implementation of enclosed geometry}

Initialization time 10 fish position coordinates and angle are arbitrary, contestants want to place a geometric shape circle at this moment, so the contestants just need to find out the distance each target fish recently, and make it to the nearest point, to complete the modelling. Of course, there are many teams have adopted a fish to swim to a certain point, it will not only reduce the amount of code, also easy to control. However, because the game requires completion within 5 minutes, each time using the nearest fish to swim to the point, no doubt greatly reduce the time of each action, but also increase the number of movements, which is more conducive to winning the game.

The author used the greedy algorithm to put the initial state into circle. First, design the location of the circle coordinate diagram, depending on the platform of Fish Setting (shown in Fig.4). The author draw the circle target coordinates, as shown in Fig.5. Then select in the round top target point_1, then use a loop to put all the distance between fish and target point_1 in custom array arr [], next use the sorting algorithm from the arr [] in ascending order, according to the results of the sorting, it can be aware that the data stored in arr[0] is the nearest distance point_1. At this time it should be known which fish corresponding to the shortest distance, and then let the fish swim to the target point point_1. So use a loop again, compare the distance between each fish of point_1 with the shortest distance, if equal, call the point-to-point implementation of the strategy, make the fish swim to point_1, and using the same strategy with the other fish. Particularly, because the fish has set a target point_1, so before 
ordering the arr[] with the distance of the target point, put the distances of the target point_2 and the fish which swam to point_1 to maximum.

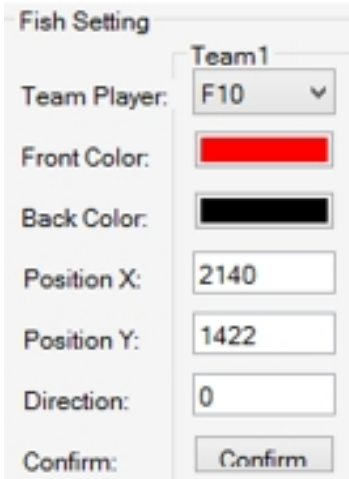

Fig.4 Fish Setting

\begin{tabular}{|l|l|l|l|}
\hline Fish & Point $X$ & Point $Y$ & Direction \\
\hline F2 & 0 & -600 & 0 \\
\hline F3 & 350 & -300 & 45 \\
\hline F4 & 450 & 150 & 90 \\
\hline F5 & 200 & 500 & 135 \\
\hline F6 & -250 & 600 & 180 \\
\hline F7 & -600 & 400 & -135 \\
\hline F8 & -700 & -50 & -90 \\
\hline F9 & -450 & -450 & -45 \\
\hline
\end{tabular}

Fig. 5 The location of the circle coordinate graph

Fig.6-1 to Fig.6-6 given the realization of the closed geometry of the circle, it takes about 30 seconds from the initial state to the target state.

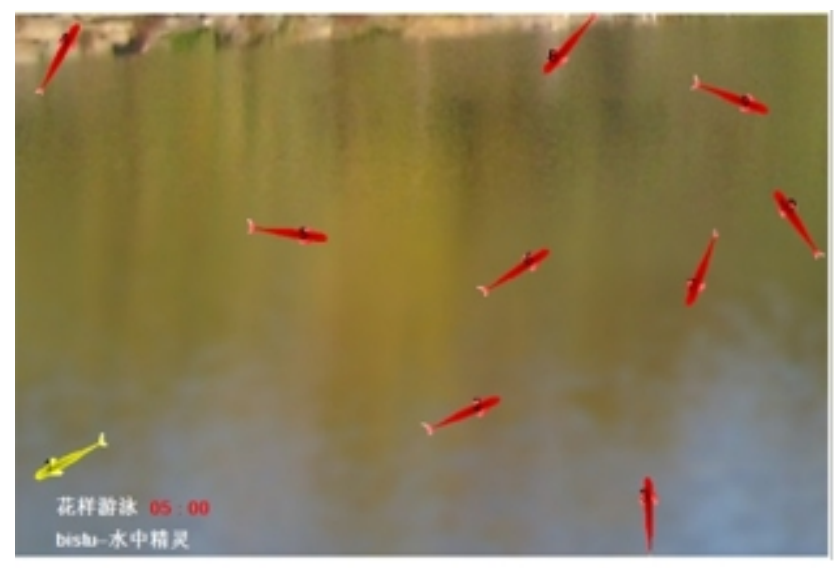

Fig.6-1 05:00 moment random

Coordinates and angles of fish

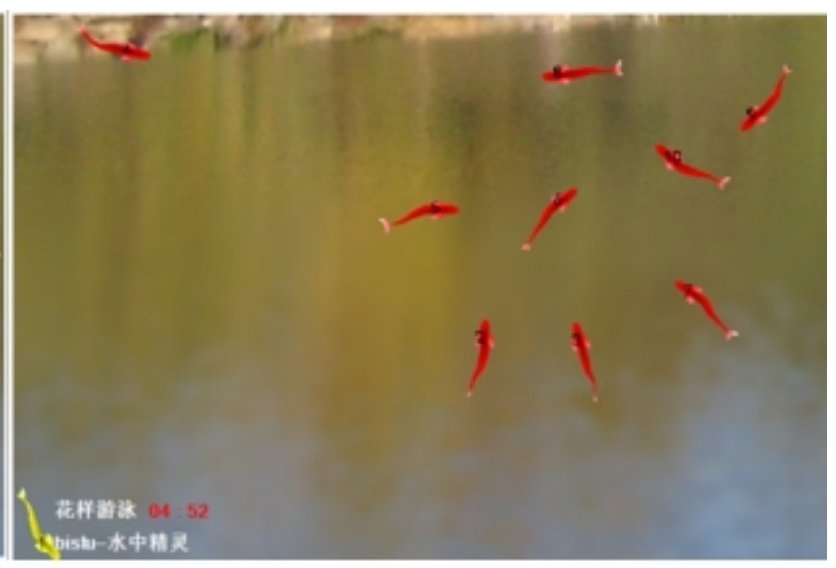

Fig.6-2 04:52 moment fish judgment target and swim to the target point

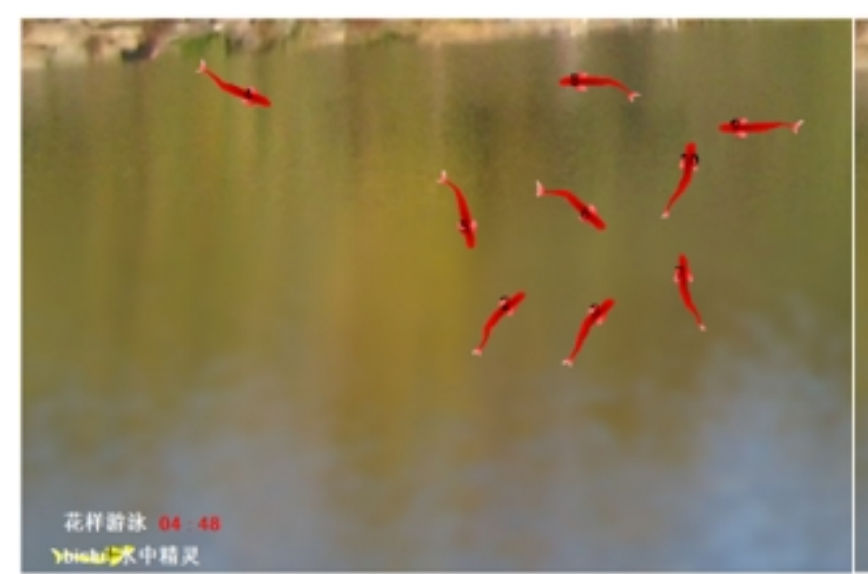

Fig.6-3 04:48 6-3 moment fish state

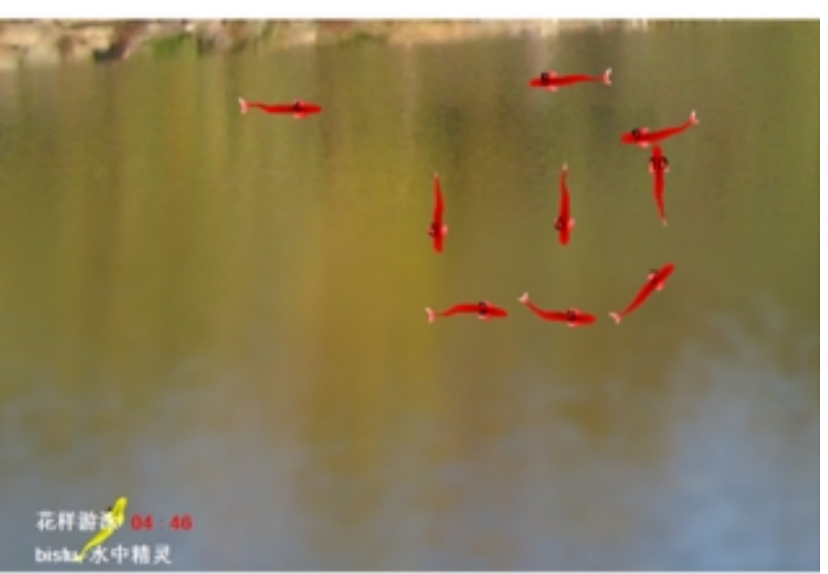

Fig.6-4 04:46 moment fish state 


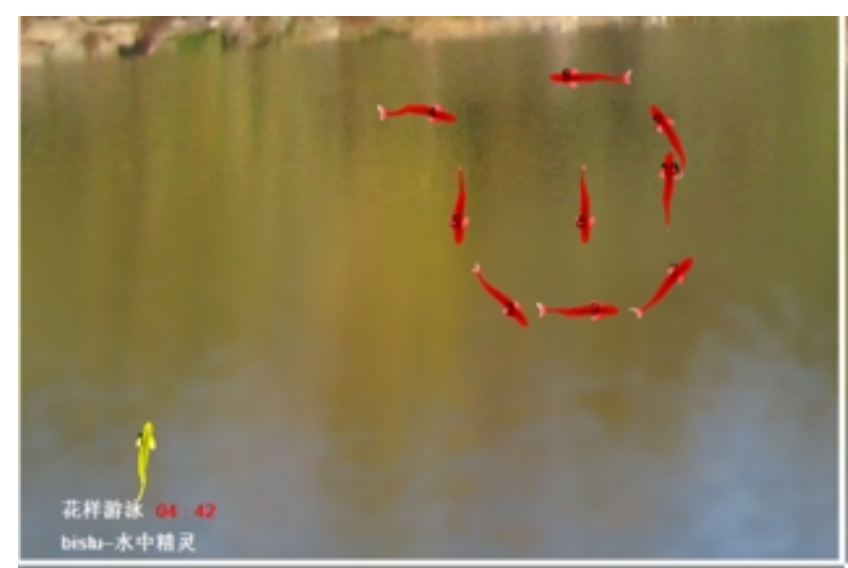

Fig.6-5 04:42 preliminary form of Geometric graphics circle

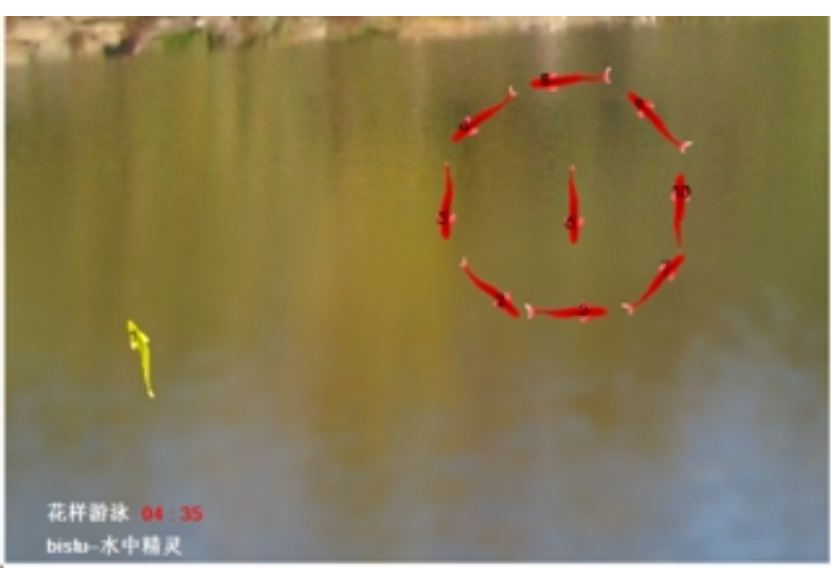

Fig.6-6 04:35 completed the geometry of the circle

\section{Implementation of keeping all the fish shape and same action}

Contest rules required to maintain the same shape and movement of fish more than five seconds. Generally use swimming straight and swing tail or other means to complete this action. Here the author realized that all fish rotation movement as a whole.

When the geometric shape of Fig.6-6 is finished, specify the appropriate angular velocity and linear velocity of each fish, so that they can rotate in a circle as a whole. It must be pointed out that if let every fish in the same action and modelling round clockwise to $1 / 2 \pi$, it must be ensure that set the linear velocity and angular when the fish swim forward radius length, the head rotation 1 / 2PI. In order to ensure that the fish swimming more than 5 seconds, you need to set the radian of the fish. Note that if the radian is judging the state of the fish, to keep the fish swam the radian of less than $2 \pi$, once beyond $2 \pi$, simple in arc system, there is no way to judge is the first lap of a state or a state of the second lap. If must exceed $2 \pi$, it can use the system function 'mission. CommonPara. TotalSeconds' to control the number of turns. The program flow chart shown in Fig.7.

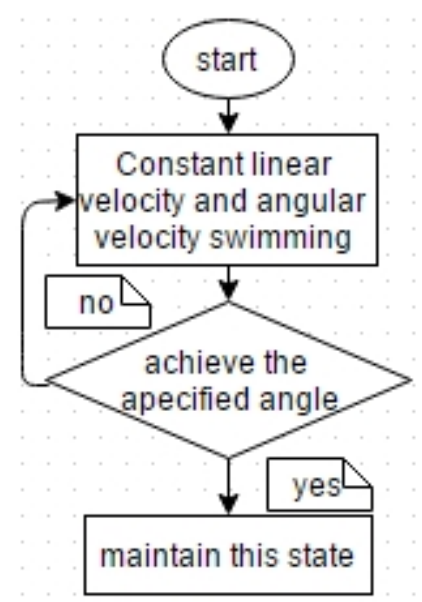

Fig. 7 program flow chart

Fig.8-1 to Fig.8-3 performance the implementation of the process of the overall clockwise $(\pi / 2)$. 


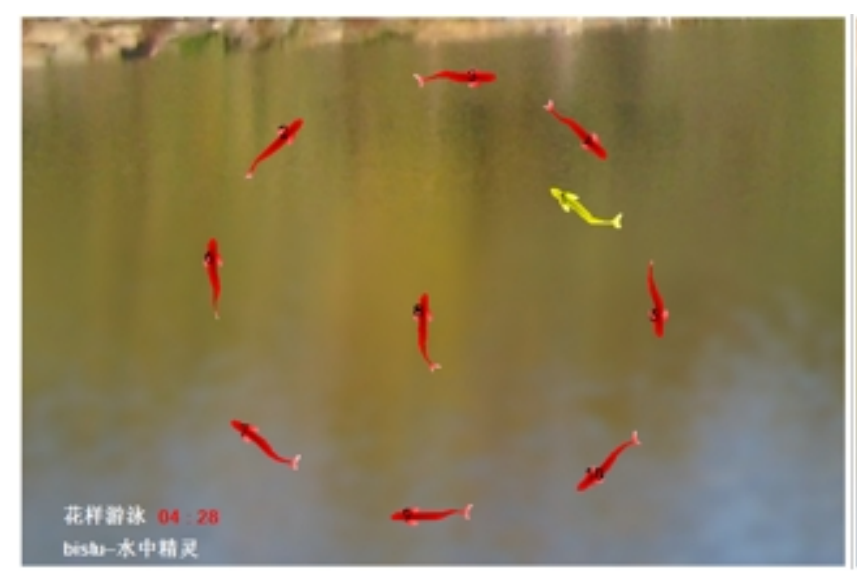

Fig. 8-1 04:28 moment just form the round shape

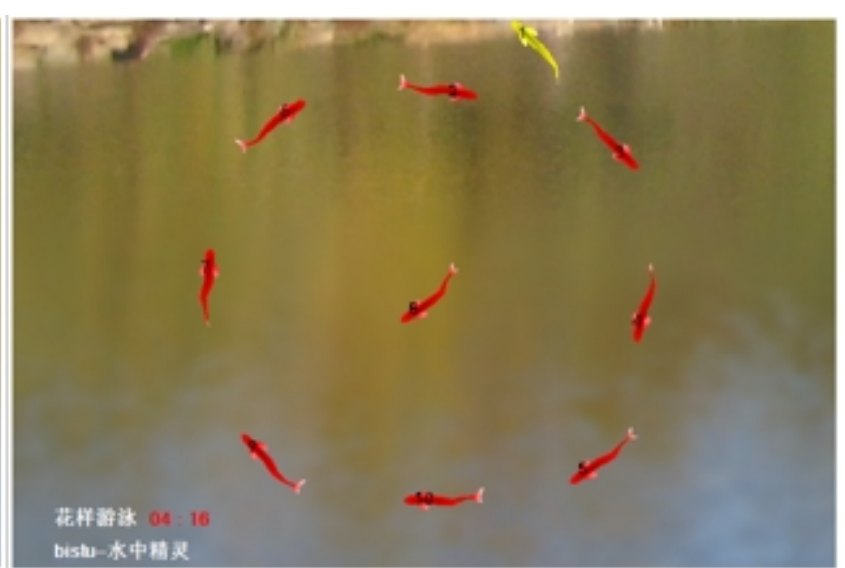

Fig. 8-2 04:16 moment whole rotate 45 degrees

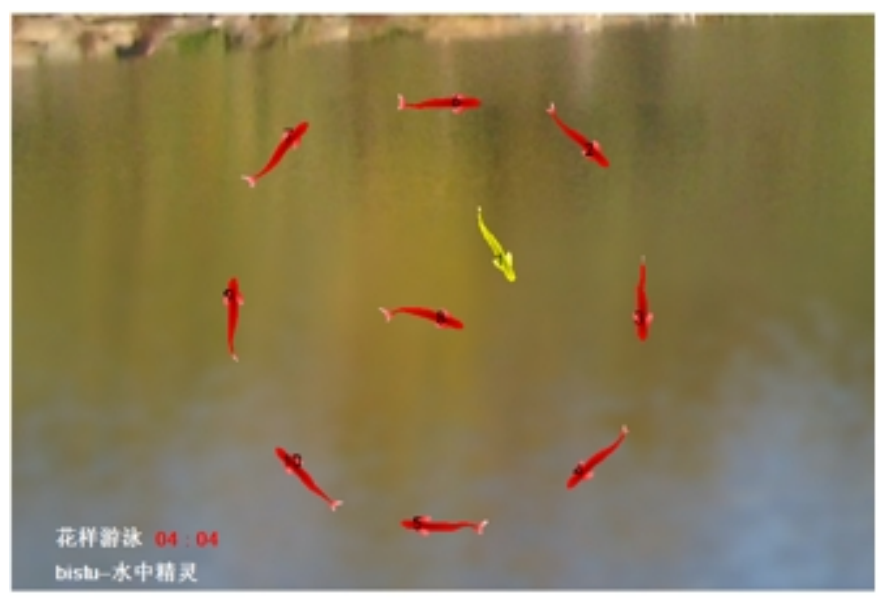

Fig. $8-3$ round whole rotated 90 degrees

\section{Implementation of static action}

Competition rules require there are 5 seconds between two shapes of the picture is still, here are two strategies to achieve. One is to use the system function "mission.CommonParam.TotalSeconds ()" to the timing, the other one is to set the local variable "I" in the loop. It can be measured the actual value of a second "I", and then we can calculate how much is the value of the "I" in 5 seconds. When reaching the 5 seconds interval, set the state value is true, and execute the next action.

Noted here, sometimes we want all the fish finished their first action, and then to the next one. There is a situation, all the fish have reached the target state, except one disturbed by yellow croaker fish. Because the conditions on the next action is all the fish have finished the specified state, so the other eight will wait for the disturbed one to complete the specified action. If the fish cannot reach the specified state or reach the specified for a long time, the next action may be not conducted or waste time. Therefore, the time limit can be added to the last action or the next action, so that it can avoid the occurrence of waiting for a long time.

As shown in Fig.9-1, by setting the local variable "I" in the loop body, it can be seen within five seconds only the yellow fish in swimming, others red fish are at rest. Five seconds after, the effect shown in Fig.9-2. 


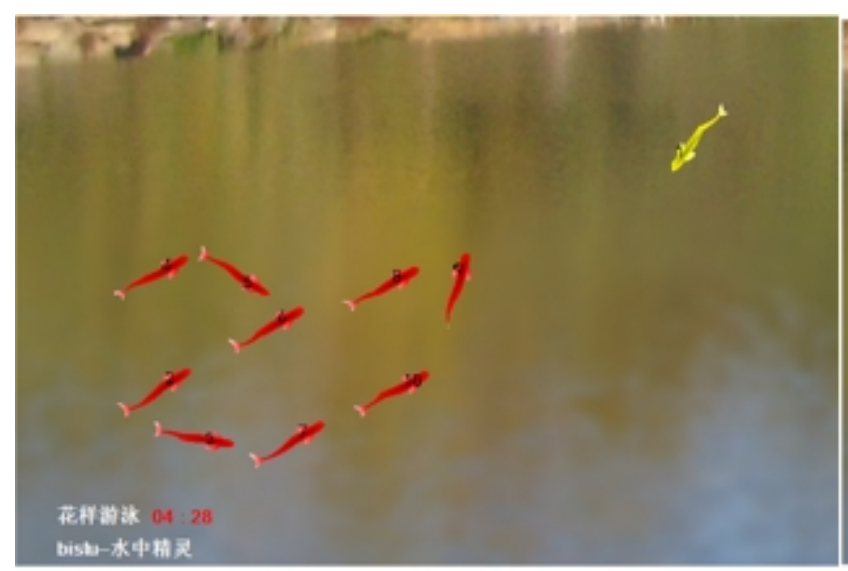

Fig.9-1 04:28 moment just a boat

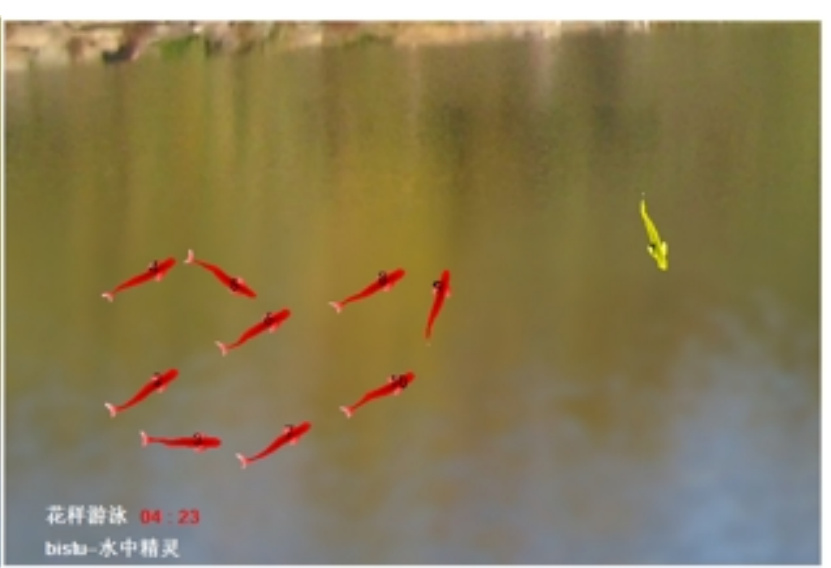

Fig. $9-25$ seconds after

\section{Other beautiful modelling}

The key to synchronized swimming events is to perfect transformation between beautiful modelling and modelling, which requires teams of brainstorming. Here the author give a few classic style race in the calendar year, as shown in Fig.10-1 and Fig.10-2.

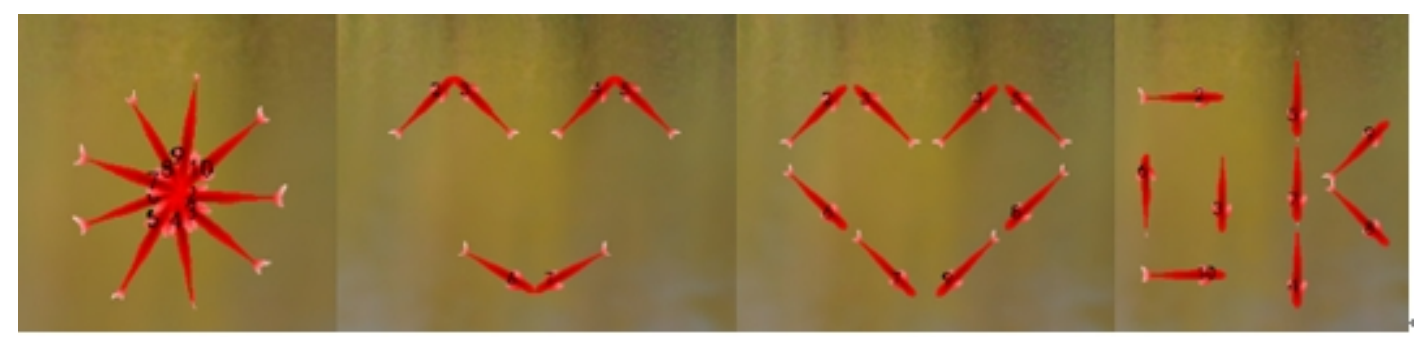

Fig.10-1 model example 1

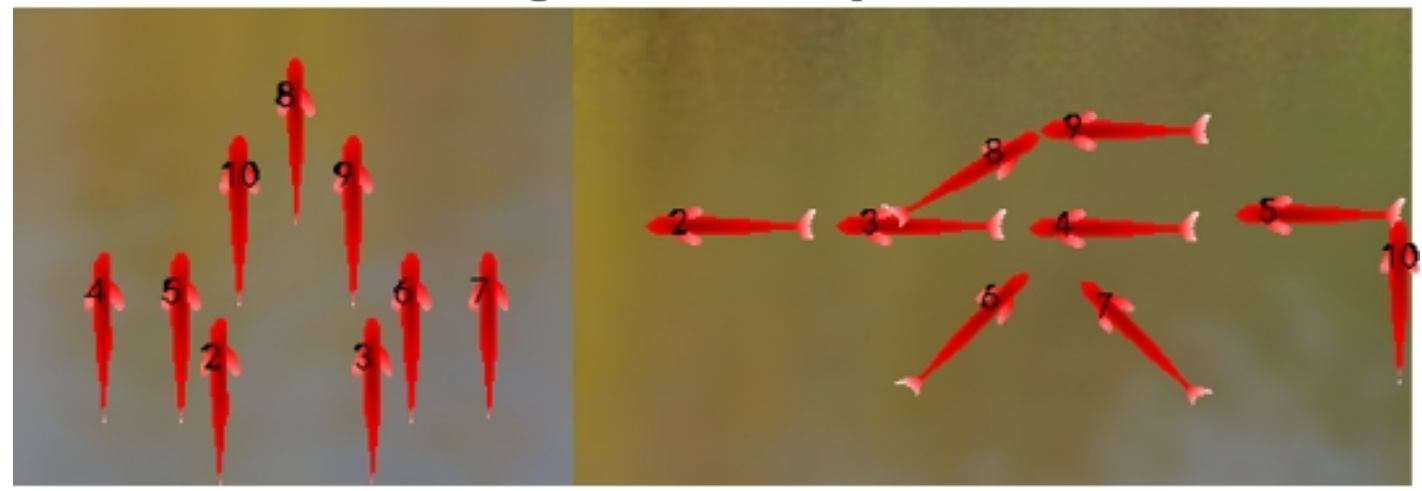

Fig. 10-2 model example 2

\section{Conclusion}

This article mainly aimed at synchronized swimming latest game rules are analyzed, and gives the implementation of corresponding action. The experimental results show that with the strategy, 9 fish can be well maintained the integrity and consistency of modeling in synchronized swimming. While there are yellow croaker, but overall remain for the realization of the Arabic numerals modelling of 2, geometry, closed circle, keep all fish the same shape and movement form for more than 5 seconds, 5 seconds between two modellings.

Using the rules of the game analysis and strategy implementation, Beijing information science and technology university Water Wizard team has achieved the first prize, in the synchronized swimming robot competition on July 24th, 2015, which prove the effectiveness of the proposed strategy. At present in this paper, the application will often appear the situation that with the yellow croaker the red 
fish cannot finish the action accurately, therefore, how to avoid yellow croaker will become the next research focus.

\section{Acknowledgements}

This paper is jointly sponsored by the Education and Teaching Project for undergraduate scientific research plan of Education Committee of the Beijing Municipality. (PXM2015_014224_000050), by Opening Project of Beijing Key Laboratory of Internet Culture and Digital Dissemination Research (ICDD201507).

\section{Reference}

[1]Xie Guang Ming. The water robot contest to promote books [M]. BeiJin: Peking University institute of technology, 2009: 1-5.

[2] http://www.ilur.org

[3]Bao Hua, Li Shu-qin, Guo Ting-ting, URWPGSim2D Ddesign and realization of synchronized swimming of URWPGSim2D, Beijing information science and technology university newspaper 。 $2011,26(5): 84-88$

[4]Liu Tiantian, Wang jiankun, Chen yanjun, Synchronized Swimming Algorithm Based on Conditions Decision [J] Ordnance Industry Automation，2014，33（12） : 90-92

[5]Chen Wei, The Research of Locomotion Modal and Game

Strategy of Robot-Fish [D]. Guangxi Technical College, 2012

[6]An Yong Yue, Li Shu Qin, Long Hainan, etc. Robotic fish simulation water polo snooker competition strategy [J]. Ordnance Industry Automation, 2012, 31 (11): 52-54. 